\title{
Turbulent flow effects in a slickwater fracture propagation in permeable rock
}

\author{
E.A. Kanin ${ }^{1^{*}}$, D.I. Garagash ${ }^{2,1}$, and A.A. Osiptsov ${ }^{1}$ \\ ${ }^{1}$ Multiphase Systems Lab, Skolkovo Institute of Science and Technology (Skoltech), \\ 3 Nobel Street, Skolkovo Innovation Center, Moscow 121205, Russian Federation \\ ${ }^{2}$ Department of Civil and Resource Engineering, Dalhousie University, 1360 \\ Barrington Street, Halifax, Nova Scotia B3H 4R2, Canada \\ *Corresponding author: evgenii.kanin@skoltech.ru
}

\begin{abstract}
This work is devoted to an analysis of the near-tip region of a hydraulic fracture driven by slickwater in a permeable saturated rock. We consider a steady-state problem of a semi-infinite fracture propagating with constant velocity. The host rock is elastic and homogeneous, and fracture propagates according to linear elastic fracture mechanics. The fluid exchange between the fracture and reservoir is governed by Carter's law. The distinguishing feature of the model is an account for the transition of the flow regime inside the crack channel from laminar to turbulent moving away from the fracture front. The main objective is to analyse the influence of the leak-off process on the laminar-to-turbulent transition and, thus, potential prominence of turbulent flow effects. Hydraulic fracturing fluid is water with polymeric additives (slickwater). These additives reduce viscous friction resulting in the decrease of energy consumption required for pumping. Compared to water, the slickwater exhibits significantly delayed transition to the turbulent regime described by the maximum drag reduction asymptote (Virk, 1975). The system of governing equations, which consists of elasticity equation, propagation condition, the continuity equation for viscous incompressible Newtonian fluid, and Poiseuille's law modified for the turbulent flow regime, is solved for the fracture aperture and fluid pressure along the fracture as a function of problem parameters. We find out that the leak-off process enhances the turbulent flow effects by shifting the transition between laminar and turbulent flow regimes closer to the fracture front, as compared to the zero-leak-off case (Lecampion \& Zia, 2019), resulting in a broader region of the fracture hosting turbulent flow. Consequently, in the permeable reservoir case, the transition to turbulent flow can be realised at a distance from the front smaller than the typical field hydraulic fracture size (10 - 100 meters). We compare the fracture width profiles with the impermeable rock case and reveal that the fracture volume increases when leak-off occurs. We analyse the problem parametric space where five limiting regimes are identified: toughness, laminar-viscosity and -leak-off, turbulent-viscosity and -leak-off. We derive analytical expressions for the fracture width and pressure profiles in the turbulent-leak-off regime while others have been established previously. By comparing the limiting solutions with the general numerical solution, we can define their applicability domains and corresponding solution regime maps. The toughness and turbulent-viscosity regimes approximate the general solution in the near- and far-fields, while the other three limiting cases can emerge in the intermediate field.
\end{abstract}




\section{Introduction}

Hydraulic fracturing is wide-spread technology utilised for the development of oil and gas reservoirs to increase production of hydrocarbons (Economides et al., 1989, 2002). Hydraulic fractures (HFs) are created via the injection of the high-pressured fluid from the Earth's surface into the formation. The injection leads to opening and growth of a tensile (mode I) fracture against the formation far field confining stress. The usage of HFs is particularly crucial for the deposits with low permeability and porosity, and such reservoirs are developed by using the multistage fractured horizontal wells with dozens of HFs. In order to ensure a sufficient fracture aperture for the subsequent proppant placement, it is required to inject hydraulic fracturing fluid at a very high rate leading to the high velocity of the fluid flow inside the crack channel. In the case of water-based hydraulic fracturing fluid with relatively low viscosity $(1-10 \mathrm{cP})$, Reynolds number of the fluid flow could become higher than the critical value corresponding to the transition of the flow regime from laminar to turbulent. As a result, a fracture region adjacent to the injection point is occupied by turbulent flow while along the remaining part the laminar flow regime is expected. Since the fracture aperture is zero at the tip, laminar flow is always realised near the fracture front.

In order to limit the energy consumption required for the fluid injection into the reservoir and, consequently, operational costs, specific polymer substances (also known as drag reduction agents) are added to pure water forming a mixture called slickwater. These additives increase the fluid viscosity and significantly decrease the friction up to $70 \%$ relatively to the original solvent (Nieuwstadt et al., 2016). The experimental work carried out by Virk $(1971,1975)$ devoted to the investigation of the turbulent pipe flow of distilled water with polymeric additives reveals the reduction of the friction factor for different polymers with different concentration when a specific value of Reynolds number is exceeded (depending on pipe diameter, concentration value). Moreover, all the experimental curves (friction factor depending on Reynolds number) reach an asymptotic behaviour called 'maximum drag reduction' (MDR) asymptote or Virk's asymptote. This asymptote can be achieved when the relatively small amount of polymers are added into the solvent, meaning that the further concentration growth does not influence on the drag reduction (Virk, 1971, 1975).

Various physical phenomena are realised during the propagation of a hydraulic fracture: viscous fluid flow inside the fracture channel, brittle rock failure and fluid exchange between the fracture and permeable formation. Some of these processes may occur at lengthscales too small to be resolved by typical computational mesh which allows performing calculations effectively in terms of the computational time (Detournay, 2016); however, they may still have a measurable impact onto the fracture propagation. In order to solve this issue, the specific near-tip region models are utilised inside the finite fracture models, e.g. KGD, penny-shaped, Planar3D. The fracture tip model plays the role of a propagation criterion determining the local propagation velocity of the crack front in each time moment. This model also allows describing the tip zone accurately: it provides the fracture width, pressure and cumulative fluid exchange volume profiles along the tip element. Various fracture tip models have been developed over time. The majority of the near-tip region models include a viscous Newtonian fluid flowing inside the fracture channel according to Poiseuille's law; the ambient reservoir can be impermeable or permeable. The fully fluid-filled HF propagating in an impermeable reservoir is discussed by Spence and Sharp (1985); Lister (1990) and Desroches et al. (1994), and the effects connected with a fluid lag are taken into account in works (Rubin, 1993; Garagash and Detournay, 2000). In a permeable reservoir case, the fluid exchange process has an impact on the fracture tip characteristics: the leak-off process governed by Carter's law is analysed by (Lenoach, 1995) and (Garagash et al., 2011), and more complex effects associated with the pore pressure diffusion and poroelasticity are described by Detournay and Garagash (2003); Kovalyshen (2010); Kanin et al. (2020). There are also several works devoted to the exploration of the non-Newtonian rheology of the HF fluid (Moukhtari and Lecampion, 2018; Dontsov and Kresse, 2018; Bessmertnykh and 
Dontsov, 2019), the cohesive zone presence and constraints of the linear elastic fracture mechanics usage in hydraulic fracturing (Garagash, 2019), and finally, the transition of the fluid flow inside the crack channel from the laminar regime to turbulent (Dontsov, 2016b; Lecampion and Zia, 2019).

In the present paper, we examine the near-tip region of a hydraulic fracture propagating in a permeable reservoir. The fluid exchange process between the fracture and ambient porous media is governed by Carter's law. We also take into account the possible transfer of the flow regime from laminar to turbulent at some distance from the fracture front where Reynolds number of the fluid flow inside the crack becomes higher than the critical value. The hydraulic fracturing fluid in our model is slickwater (pure water solvent with polymeric additives), and we assume that the MDR asymptote governs its friction factor during the turbulent flow regime. For the problem formulation, we will rely on the original model framework of Lecampion and Zia (2019), where the authors investigate a similar problem in the case of an impermeable reservoir. In the current work, we analyse how the presence of the leak-off process affects the transition to the turbulent flow regimes. A fracture tip model with Carter's leak-off and laminar-turbulent flow transition has been previously studied by Dontsov (2016b) for the choice of pure water as the fracturing fluid. However, the flow behaviour of slickwater is significantly different from that of pure water. Firstly, the water-based fluid with polymeric additives has much less friction, secondly, the laminar-to-turbulent transition takes place at smaller Reynolds number (the exact values will be presented further) meaning that the transient/fully turbulent flow regime occurs along the broader region of the crack in the case of slickwater, and, thirdly, the turbulent regime for slickwater flow is characterised by the distinct MDR behaviour not presented in pure water turbulent flow.

This paper is organised as follows. Firstly, we formulate the problem, enumerate the assumptions, and write out the system of governing equations. Secondly, we outline the limiting propagation regimes also know as vertex solutions. Thirdly, we present particular solution profiles for the fracture opening and fluid pressure and carry out a comparison with an impermeable reservoir case (Lecampion and Zia, 2019). Finally, we analyse the parametric space of the problem by constructing the regime maps showing the applicability domains of the limiting solutions and examine the behaviour of the transition boundary between the flow regimes.

\section{Model formulation}

\subsection{Problem formulation}

Near-tip region of a fluid-driven fracture is investigated with the help of a model for a semi-infinite plane strain fracture propagating with constant velocity $V$ which is interpreted as the instantaneous local front velocity of a finite (parent) fracture. A schematic picture of the discussed model is presented in figure 1. Since the propagation velocity is constant, it is possible to introduce moving coordinate $x$ denoting distance from the moving fracture front, and in this coordinate system, the problem is steady-state.

The ambient rock is taken as a linear-elastic described by Young's modulus $E$ and Poisson's ratio $\nu$. The length scale of the region ahead of the propagating fracture front where the rock failure takes place is assumed to be small compared to other length scales realised in the model, e.g., the ones connected with the fluid viscosity and leak-off. Therefore, the linear elastic fracture mechanics (LEFM) theory is applied to model the quasi-static propagation of the fracture in the solid media with toughness $K_{I c}$.

The fracture faces are loaded internally by the fluid pressure $p_{f}(x)$ while the rock is subjected to the far-field confining stress $\sigma_{o}$. The net pressure function $p(x)=p_{f}(x)-\sigma_{o}$ and the aperture profile $w(x)$ completely characterise a semi-infinite fracture solution. The HF fluid is slickwater which can 


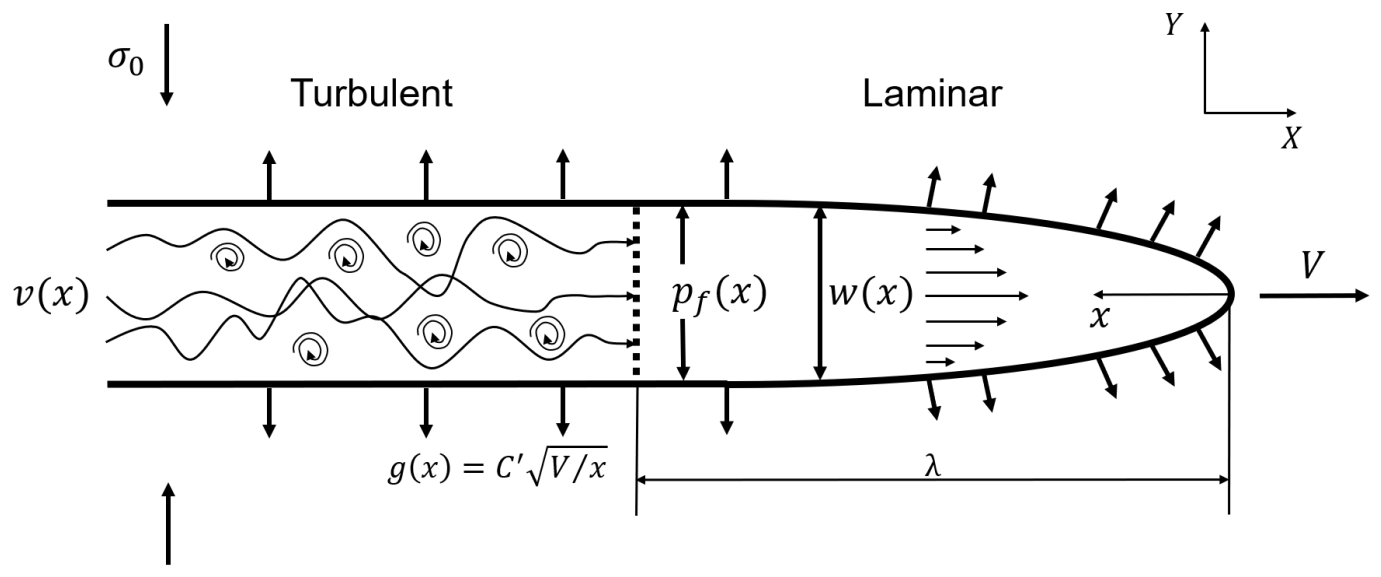

Figure 1: Schematics of the fracture-tip model with turbulent flow and leak-off.

be characterised as Newtonian liquid with viscosity $\mu$ and density $\rho$ in the laminar flow regime, while the rheological response from the onset of the transition to turbulent flow is discussed in details in the next section. The fluid flow inside the fracture channel is described by the lubrication theory (Batchelor, 1967). Let us define Reynolds number for the channel flow as $R e=\rho v w / \mu$, where $v$ is the fluid velocity. Reynolds number grows from 0 to infinity if we move away from the tip. Let us suppose that $R e$ is less than the critical value $R e_{c}$ along the fracture region $x \in(0, \lambda)$ (figure 1) resulting in the laminar flow regime there. The onset of the flow regime transition to turbulence is located at point $x=\lambda$, and the flow regime along the domain $x \in(\lambda,+\infty)$ is non-laminar (from transient to fully-turbulent). In the model, the flow regime transition is captured via the friction factor $f$ usage. The fluid exchange between the host rock with porosity $\phi$ and permeability $k$, and the fracture is taken in the form of Carter's law (Carter, 1957) implying the fluid exchange rate to be proportional to the inverted square root of the 'exposure' time (the interval between the current time and the moment when the fracture front, assumed to coincide with the fluid front, reaches the considered point of the fracture plane). The proportionality coefficient $C_{L}$, or Carter's leak-off coefficient, can be expressed as: $C_{L}=k\left(\sigma_{o}-p_{o}\right) /(\mu \sqrt{\pi c})$, where $c=k /\left(\phi c_{t} \mu\right)$ is the diffusivity coefficient $\left(c_{t}\right.$ is the fluid compressibility) and $p_{o}$ is the far-field pore pressure, when the cake-building (deposition of the polymer additives onto the fracture walls) is neglected, and formation fluid has similar properties to that of the fracturing fluid in the context of the flow through the porous rock (Kovalyshen, 2010; Kanin et al., 2020).

\subsection{Governing equations}

Firstly, we introduce the moving coordinate system $(x, y)$ linked with the fixed coordinates $(X, Y)$ by the relations: $x=V t-X, y=Y$; the problem is stationary in the coordinates $(x, y)$. The system of governing equations is formulated for unknown opening $w(x)$ and net fluid pressure $p(x)$ profiles with distance $x$ from the moving tip, and the set of material parameters:

$$
E^{\prime}=\frac{E}{1-\nu^{2}}, \quad K^{\prime}=4 \sqrt{\frac{2}{\pi}} K_{I c}, \quad \mu^{\prime}=12 \mu, \quad C^{\prime}=2 C_{L},
$$

where $E^{\prime}$ is the plane strain elastic modulus, $K^{\prime}$ and $\mu^{\prime}$ are the toughness and viscosity parameters, and $C^{\prime}$ is the leak-off parameter.

We begin with the fracture propagation equation based on the LEFM. Fracture propagates when the stress intensity factor at the tip equals to the rock toughness, e.g., (Kanninen and Popelar, 1985): $K_{I}=K_{I c}$. This condition can be equivalently written in the form of the fracture opening asymptotic 
behaviour near the tip (Irvin, 1957):

$$
w=\frac{K^{\prime}}{E^{\prime}} \sqrt{x}, \quad x \rightarrow 0 .
$$

Further, we move on to the elasticity equation according to which the net fluid pressure $p(x)$ can be expressed through the aperture profile $w(x)$ (Bilby and Eshelby, 1968):

$$
p(x)=\frac{E^{\prime}}{4 \pi} \int_{0}^{\infty} \frac{d w}{d s} \frac{d s}{x-s} .
$$

Next, we consider the fluid flow inside the fracture channel. For this purpose, we utilise the continuity equation averaged across the fracture aperture for an incompressible fluid. In the moving coordinate system, it can be written in the following form:

$$
V \frac{d w}{d x}-\frac{d(w v)}{d x}+g=0
$$

where $g$ is the leak-off rate governed by Carter's law (Carter, 1957):

$$
g=C^{\prime} \sqrt{\frac{V}{x}} .
$$

Substituting equation (5) into (4) and integrating from the tip $(x=0)$ to some coordinate $x$, we obtain

$$
v=V+2 C^{\prime} \frac{\sqrt{V x}}{w}
$$

During the integration we take into account the boundary conditions: $w(0)=0$ and $\left.(w v)\right|_{x=0}=$ 0 .

Finally, we should consider the width-averaged momentum conservation equation:

$$
v^{2}=\frac{w}{\rho f} \frac{d p}{d x}
$$

where $f$ is the Fanning friction factor.

In the case of the laminar flow regime, equation (7) has the form of Poiseuille's law:

$$
v=\frac{w^{2}}{\mu^{\prime}} \frac{d p}{d x}
$$

By comparing equations (7) and (8), one can obtain the friction factor expression for the laminar flow regime: $f^{\text {lam }}=12 / R e$. In turn, the Fanning friction factor for pipe laminar flow equals $f_{d}^{\text {lam }}=$ $16 / R e_{d}$, where $R e_{d}=\rho v d / \mu$ is Reynolds number for pipe flow suggesting $R e_{d}=4 / 3 \cdot R e$. This formula is essential for translating the friction factor curves established for the pipe flow, e.g., see (Brill and Mukherjee, 1999)), to the case of the channel flow (in the fracture), i.e. $f=f\left(R e_{d}\right)=$ $f(4 / 3 \cdot R e)$ (Lecampion and Zia, 2019). Further, we introduce the normalised friction factor $\tilde{f}=$ $f / f^{\text {lam }}$ (Dontsov, 2016b) and rewrite equation (7):

$$
v=\frac{w^{2}}{\mu^{\prime} \tilde{f}} \frac{d p}{d x},
$$

We have already described the flow behaviour during the laminar flow regime. Now, we move on to the discussion of turbulent flow. Since the HF fluid is slickwater, we consider the friction factor $f$ 

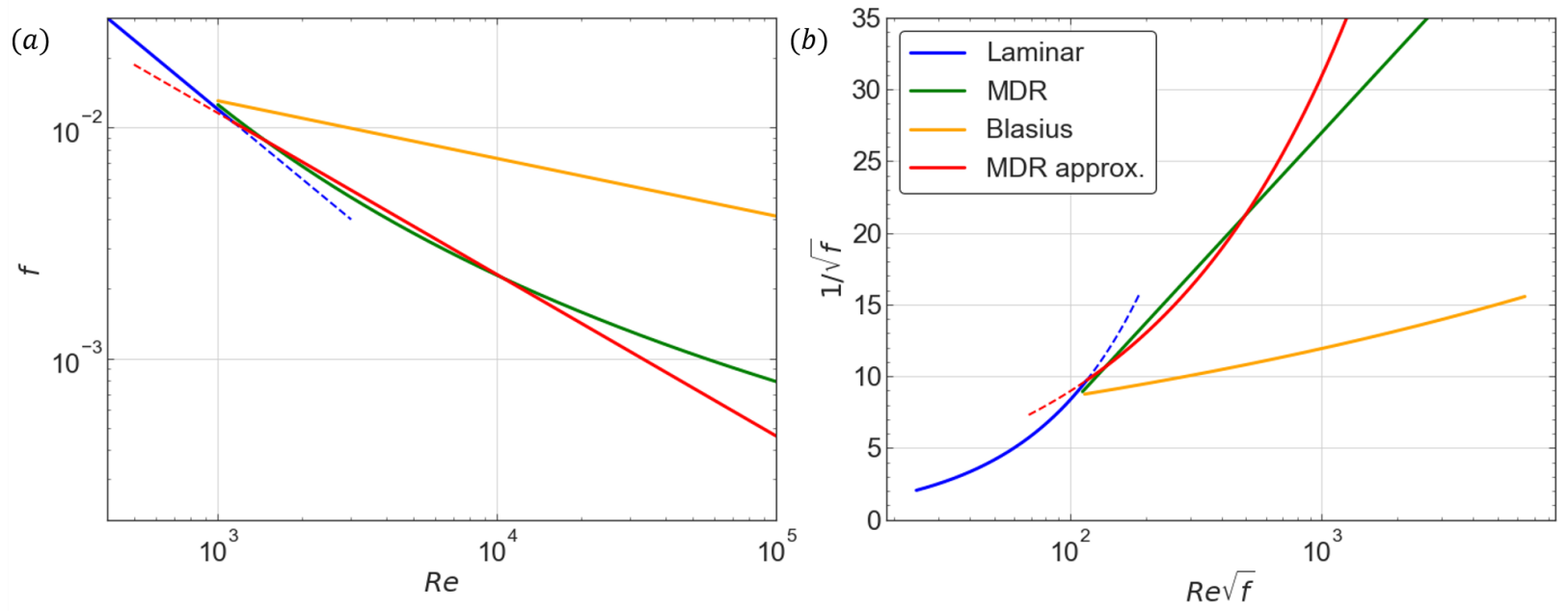

Figure 2: Friction factor depending on Reynolds number in ordinary (a) and Prandtl-Karman (b) coordinates. The laminar branch is shown by blue colour, MDR asymptote (10) and its approximation (11) are depicted by solid green and red lines, correspondingly. Blasius asymptote (pure water, smooth walls) is plotted by solid orange line. Dashed lines present functions continuation beyond the intersection point between laminar and MDR simplified curves.

governed by the MDR asymptote: a phenomenological relation proposed by Virk (1971, 1975), when $R e>R e_{c}$ (non-laminar flow):

$$
\frac{1}{\sqrt{f}}=19 \log _{10}\left(R e_{d} \sqrt{f}\right)-32.4
$$

To facilitate the solution of the HF tip problem, we will utilise a power-law approximation of (10) proposed by Lecampion and Zia (2019):

$$
f=f_{0} R e_{d}^{-n}=f_{0}^{\prime} R e^{-n},
$$

where $f_{0}=1.78, n=0.7$ and $f_{0}^{\prime}=f_{0}(4 / 3)^{-n}=1.46$.

Figure 2 presents the function $f(R e)$ during laminar and turbulent regimes in traditional (a) and Prandtl-Karman (b) coordinates. Apart from the MDR asymptote (green line), we also show Blasius asymptote for turbulent pipe flow (smooth walls case) of pure water adjusted to the channel geometry in order to demonstrate quantitatively how the slickwater drag reduction agents decrease friction. The MDR approximation (11) closely approximates Virk's asymptote (10) within the range $R e \in\left(10^{3}, 1.5 \cdot 10^{4}\right)$ with the relative deviation less than $5 \%$.

Further, we define the friction factor function for the whole range of Reynolds numbers, i.e. from the laminar regime to the turbulent one, similar to Lecampion and Zia (2019):

$$
f= \begin{cases}12 / R e, & R e \leq R e_{c}, \\ f_{0}^{\prime} R e^{-n}, & R e>R e_{c},\end{cases}
$$

where $R e_{c}$ is the critical Reynolds number for slickwater case. The critical value is defined as an intersection of the laminar and turbulent (equation (11)) branches in order to ensure the function $f(R e)$ continuity: $R e_{c}=\left(12 / f_{0}^{\prime}\right)^{1 /(1-n)}=1132.6$. The defined $f(R e)$ by equation (12) is shown in figure 2 by the combination of blue (laminar part) and red (turbulent part) solid lines.

Similarly to equation (12), we write out the expression for the scaled friction factor $\tilde{f}$ :

$$
\tilde{f}= \begin{cases}1, & R e \leq R e_{c} \\ f_{0}^{\prime \prime} \cdot R e^{1-n}, & R e>R e_{c}\end{cases}
$$


where $f_{0}^{\prime \prime}=f_{0}^{\prime} / 12=0.122$.

Finally, one can compare the critical Reynolds number value for slickwater with the pure water case. It is known that the discussed value for the pipe flow is approximately $R e_{d}^{c} \approx 2100$ meaning that for channel flow it is equal to $R e^{c}=3 / 4 \cdot R e_{d}^{c} \approx 1575$. As a result, the laminar-to-turbulent transition for the slickwater occurs at smaller Reynolds number.

\section{Limiting propagation regimes of a semi-infinite fracture with leak- off and laminar/turbulent flow}

It is known that two different mechanisms govern the propagation regime of a finite hydraulic fracture (see review of Detournay (2016) and references there in). The first one is concerned with the distribution of the total dissipated energy between the creation of new fracture surfaces (toughness) and viscous fluid flow inside the fracture channel (viscosity). The second mechanism is related to the distribution of the total injected fluid between the fracture (storage) and the reservoir (leakoff). During fracture propagation, the partitioning of the dissipated energy and injected fluid changes over time, resulting in the realisation of various limiting propagation regimes dominated by toughness/viscosity and storage/leak-off mechanisms. E.g., in the case of penny-shaped/KGD cracks these regimes (also called as vertex solutions) are described by Savitski and Detournay (2002); Bunger et al. (2005); Garagash (2006); Adachi and Detournay (2008), and their applicability domains are determined by Madyarova (2003); Hu and Garagash (2010) and Dontsov (2016a, 2017). The similar concept can be applied when we study a semi-infinite fracture propagating with constant velocity, e.g., Garagash et al. (2011), in which case the partitions of the dissipated energy and that of the injected fluid evolve with the distance for the moving fracture tip.

The leak-off parameter $C^{\prime}$ controls the partitioning of the injected fluid, $\mu^{\prime}$ and $K^{\prime}$ have an influence on the dissipated energy distribution, and Reynolds number indicates the occurring flow regime. Five limiting propagation regimes can be distinguished in the model: three of them are related to laminar flow and the remaining two for turbulent flow:

$k$ : toughness $\left(\mu^{\prime}=0\right)$;

$m: \quad$ laminar-storage-viscosity $\left(C^{\prime}=0, K^{\prime}=0, R e<R e_{c}\right)$;

$\tilde{m}: \quad$ laminar-leak-off-viscosity $\left(C^{\prime} \rightarrow \infty, K^{\prime}=0, R e<R e_{c}\right)$;

$t$ : $\quad$ turbulent-storage-viscosity $\left(C^{\prime}=0, K^{\prime}=0, R e>R e_{c}\right)$;

$\tilde{t}: \quad$ turbulent-leak-off-viscosity $\left(C^{\prime} \rightarrow \infty, K^{\prime}=0, R e>R e_{c}\right)$.

We begin with the laminar flow regime case: $k, m$ and $\tilde{m}$ vertices. The detailed description of this fracture tip model is provided by Garagash et al. (2011), and here we summarise the main points for the completeness. These limiting propagation regimes are the solutions for the entire semi-infinite fracture, and they can be found in the form of a monomial solution of the elasticity equation (3) (Kanninen and Popelar, 1985):

$$
w_{\lambda}(x)=B x^{\lambda}, \quad p_{\lambda}(x)=E^{\prime} B f(\lambda) x^{\lambda-1} ; \quad f(\lambda)=\lambda \cot (\pi \lambda) / 4, \quad \lambda \in(0,1),
$$

where coefficients $B$ and $\lambda$ are determined from the lubrication equation (6) with the corresponding values of governing parameters $\left(C^{\prime}, \mu^{\prime}, K^{\prime}\right)$ mentioned above. In toughness dominated regime $(k)$, the fluid viscosity can be neglected $\left(\mu^{\prime}=0\right)$ leading to zero pressure and the fracture opening profile in accordance with the propagation condition (2). In the storage-viscosity case $(m)$, the leaked-off volume and toughness are null $\left(C^{\prime}=K^{\prime}=0\right)$, and the solution can be derived by balancing the fluid flow velocity $v(8)$ with the propagation velocity $V$. In the leak-off-viscosity dominated case $(\tilde{m})$, the leaked-off volume is much larger than the stored in the fracture $\left(C^{\prime} \rightarrow \infty\right)$, and the toughness is negligible $\left(K^{\prime}=0\right)$. That is why, the $\tilde{m}$-vertex solution is found by comparing the fluid flux velocity 


\begin{tabular}{l|c|c|c|}
\multicolumn{1}{c|}{ Limiting solutions } & opening $w(x)$ & net pressure $p(x)$ & velocity $v(x)$ \\
\hline \hline $\mathbf{k}$ Toughness $\left(\mu^{\prime}=0\right)$ & $\ell_{k}^{1 / 2} x^{1 / 2}$ & 0 & $V+E^{\prime} C^{\prime} \sqrt{V} / K^{\prime}$ \\
\hline $\mathbf{m}$ Storage-viscosity $\left(K^{\prime}=C^{\prime}=0\right)$ & $\beta_{0} \ell_{m}^{1 / 3} x^{2 / 3}$ & $\delta_{0} E^{\prime}\left(\ell_{m} / x\right)^{1 / 3}$ & $V$ \\
\hline m Leak-off-viscosity $\left(K^{\prime}=0, C^{\prime} \rightarrow \infty\right)$ & $\tilde{\beta}_{0} \ell_{\tilde{m}}^{3 / 8} x^{5 / 8}$ & $\tilde{\delta}_{0} E^{\prime}\left(\ell_{\tilde{m}} / x\right)^{3 / 8}$ & $V / \tilde{\beta}_{0} \cdot\left(\ell_{\tilde{m}}^{9} /\left(\ell_{m}^{8} x\right)\right)^{1 / 8}$ \\
\hline \hline
\end{tabular}

$$
\text { Coefficients: } \beta_{0}=2^{1 / 3} 3^{5 / 6}, \delta_{0}=\beta_{0} f(2 / 3), \tilde{\beta}_{0}=2.534, \tilde{\delta}_{0}=\tilde{\beta}_{0} f(5 / 8) .
$$

Table 1: Laminar vertex solutions of a semi-infinite hydraulic fracture with Carter's leak-off.

$v$ (8) with Carter's leak-off term. All three vertex solutions are written out in table 1 through the following length scales:

$$
\ell_{k}=\left(K^{\prime} / E^{\prime}\right)^{2}, \quad \ell_{m}=V \mu^{\prime} / E^{\prime}, \quad \ell_{\tilde{m}}=\left(C^{\prime} \sqrt{V} \mu^{\prime} / E^{\prime}\right)^{2 / 3} .
$$

As it is shown in (Garagash et al., 2011), in the general case when $K^{\prime}, C^{\prime}$ and $\mu^{\prime}$ have finite nonzero values, the $k$-vertex solution is applicable in the near-field of the general solution, while $\tilde{m}$ and $m$ emerge in the intermediate (for large leak-off) and far-field correspondingly. However, when we introduce into the model the flow regime transition from laminar to turbulent, the region occupied by the laminar flow regime is bounded and locates near the fracture front (since $R e \rightarrow 0$ when $x \rightarrow 0$ ). Hence, the applicability domain of the laminar-storage-viscosity $(\mathrm{m})$ solution is expected to shift to the intermediate field.

Further, we move on to the turbulent-storage-viscosity $(t)$ and turbulent-leak-off-viscosity $(\tilde{t})$ regimes. These vertex solutions have been derived by Dontsov (2016b) for pure water case, and $t$-asymptote is presented by Lecampion and Zia (2019) for slickwater (MDR asymptote). In the further discussion, we write out the $t$-vertex solution and derive formulas for the $\tilde{t}$-vertex for Virk's asymptote.

Using the expression for the scaled friction factor $\tilde{f}$ for turbulent flow (13) and substitute it into lubrication equation (9) combined with (6), we obtain the following formula that is similar to power-law fluid case (Desroches et al., 1994):

$$
\left(V+2 C^{\prime} \frac{\sqrt{V x}}{w}\right)^{m}=\frac{w^{n+1}}{c} \frac{d p}{d x}
$$

where $m=2-n$ and $c=f_{0}^{\prime} \mu^{n} \rho^{1-n}$.

Based on the reasoning similar to that of Garagash et al. (2011) for the laminar flow case, the turbulentstorage-viscosity $(t)$ regime is expected to provide the asymptotic behaviour of the general solution in the far-field. In order to derive the analytical solution for this vertex, we should substitute the monomial solution (14) into equation (16) and balance $V^{m}$ with the right-hand-side. As a result, we obtain the vertex solution previously identified by Lecampion and Zia (2019):

$$
w_{t}=\beta_{t} \ell_{t}^{n /(n+2)} x^{2 /(n+2)}, \quad p_{t}=\delta_{t} E^{\prime}\left(\ell_{t} / x\right)^{n /(n+2)} ; \quad \ell_{t}=\left(c V^{m} / E^{\prime}\right)^{1 / n},
$$

where $\ell_{t}$ is the characteristic length scale associated with $t$-asymptote, and prefactors are given by:

$$
\beta_{t}=\left(\frac{2(n+2)^{2}}{n} \tan \left(\frac{-2 \pi}{n+2}\right)\right)^{1 /(n+2)}, \quad \delta_{t}=\beta_{n} f\left(\frac{2}{n+2}\right) .
$$

Using equations (17) and (18), one can notice that the $t$-asymptote takes a form of the $m$-vertex solution (table 1) when $n=m=1$ and $c=\mu^{\prime}$ (powers and coefficients correspond to the laminar flow regime). 
Now, we consider the turbulent-leak-off-viscosity $(\tilde{t})$ asymptote which is expected to be realised in the intermediate-field of the general solution when both turbulent and leak-off effects are large (these are to be formally quantified by corresponding non-dimensional numbers $\chi$ and $\mathcal{R}$ introduced in the next Section). To derive this limiting solution, we balance the Carter's term on the left-handside of equation (16) with its right hand side, and look for the solution in the monomial form (14) with the following result:

$$
w_{\tilde{t}}=\tilde{\beta}_{\tilde{t}} \ell_{\tilde{t}}^{(m+2 n) /(2(m+n+2))} x^{(m+4) /(2(m+n+2))}, \quad p_{\tilde{t}}=\tilde{\delta}_{\tilde{t}} E^{\prime}\left(\ell_{\tilde{t}} / x\right)^{(m+2 n) /(2(m+n+2))} ;
$$

where $\ell_{\tilde{t}}=\left(2^{m} c C^{\prime m} V^{m / 2} / E^{\prime}\right)^{2 /(m+2 n)}$ is the newly introduced length scale $\left(w_{\tilde{t}}(\tilde{t}) \sim \ell_{\tilde{t}}\right)$ and coefficients:

$$
\tilde{\beta}_{\tilde{t}}=\left(\frac{2^{4}(m+n+2)^{2}}{(m+4)(m+2 n)} \tan \left(-\frac{\pi(m+4)}{2(m+n+2)}\right)\right)^{1 /(m+n+2)} \quad, \quad \tilde{\delta}_{\tilde{t}}=\tilde{\beta}_{n} f\left(\frac{m+4}{2(m+n+2)}\right) .
$$

If we substitute values of $c, n, m$ responsible for laminar flow into equations (19), (20), we obtain $\tilde{m}$-vertex solution (table 1).

\section{Solution}

\subsection{Normalisation of governing equations}

In this section, we discuss the normalised variables and the dimensionless form of governing equations. The normalisation is required for reducing the number of problem parameters, and we will calculate the general numerical solution in the dimensionless form. We choose the $m k$-scaling proposed by Garagash et al. (2011) for which the characteristic length, width and pressure scales have the following form:

$$
\ell_{m k}=\frac{\ell_{k}^{3}}{\ell_{m}^{2}}=\frac{K^{\prime 6}}{E^{\prime 4} V^{2} \mu^{\prime 2}}, \quad w_{m k}=\frac{\ell_{k}^{2}}{\ell_{m}}=\frac{K^{\prime 4}}{E^{\prime 3} V \mu^{\prime}}, \quad p_{m k}=\frac{E^{\prime} \ell_{m}}{\ell_{k}}=\frac{E^{\prime 2} V \mu^{\prime}}{K^{\prime 2}},
$$

This scaling characterises the transition between the toughness $(k)$ and storage-viscosity $(m)$ propagation regimes in laminar flow case. Further, we introduce the normalised distance to the tip, opening and fluid net pressure, respectively,

$$
\xi=x / \ell_{m k}, \quad \Omega=w / w_{m k}, \quad \Pi=p / p_{m k}
$$

Using the set of parameters (22), we rewrite the system of governing equations in the normalised form:

- Propagation:

$$
\Omega=\sqrt{\xi}, \quad \xi \rightarrow 0
$$

- Elasticity:

$$
\Pi(\xi)=\frac{1}{4 \pi} \int_{0}^{\infty} \frac{d \Omega}{d s} \frac{d s}{\xi-s}
$$

- Lubrication:

$$
\frac{\Omega^{2}}{\tilde{f}} \frac{d \Pi}{d \xi}=1+\frac{\chi \sqrt{\xi}}{\Omega}
$$


where we introduce the dimensionless Carter's leak-off coefficient (factor of two larger than that in (Garagash et al., 2011)):

$$
\chi=\frac{2 C^{\prime} E^{\prime}}{\sqrt{V} K^{\prime}}
$$

and the normalised friction factor $\tilde{f}$ :

$$
\tilde{f}= \begin{cases}1, & \xi \leq \Lambda, \\ f_{0}^{\prime \prime} \cdot \mathcal{R}^{1-n}(\Omega+\chi \sqrt{\xi})^{1-n}, & \xi>\Lambda,\end{cases}
$$

In equation (27), we utilise the characteristic Reynolds number introduced by Lecampion and Zia (2019):

$$
\mathcal{R}=12 \rho K^{\prime 4} /\left(E^{\prime 3} \mu^{\prime 2}\right)
$$

and the notation $\Lambda(\chi, \mathcal{R})=\lambda / \ell_{m k}$ for the transition point between the laminar and the turbulent flow regimes which is a solution of the following equation:

$$
\Omega(\Lambda)+\chi \sqrt{\Lambda}=R e_{c} / \mathcal{R} .
$$

As it can be seen from the system of equations (23) - (27), the problem solution, i.e. the crack opening and net fluid pressure profiles, depends on two parameters $\chi$ and $\mathcal{R}$, and the normalised distance from the tip $\xi: \Omega(\xi, \chi, \mathcal{R})$ and $\Pi(\xi, \chi, \mathcal{R})$. The present model has two limiting cases: 1$)$ when $\chi \rightarrow 0$ it reduces to the tip model of Lecampion and Zia (2019), and 2) when $\mathcal{R} \rightarrow 0$, it has the form of Garagash et al. (2011) model.

The general solution to the problem (23) - (27) is computed numerically by using the algorithm described in (Garagash et al., 2011). This approach utilises the exact form of the near- and far-field asymptotic behaviour of the solution, and, in this case, they are toughness ( $k$ ) and turbulent-storageviscosity $(t)$ regimes. Using the $m k$-scaling (equation (22) with (21)), we write out their exact forms (since the 'zero'-order term of the pressure profile at $k$-vertex is zero, we utilise the next order term from the asymptotic expansion (Garagash et al., 2011)):

$$
\begin{aligned}
& \operatorname{Near-field~}(k): \quad \Omega_{k}=\xi^{1 / 2}, \quad \Pi_{k}=(1+\chi) \ln \left(\xi / \xi_{0}\right) \text {; } \\
& \text { Far-field }(t): \quad \Omega_{t}=\beta_{t} \xi^{2 /(n+2)} \mathcal{R}^{(1-n) /(n+2)} f_{0}^{\prime \prime 1 /(2+n)}, \Pi_{t}=\delta_{t} \xi^{-n /(n+2)} \mathcal{R}^{(1-n) /(n+2)} f_{0}^{\prime \prime} 1 /(2+n) ;
\end{aligned}
$$

where the coefficient $\xi_{0}$ is a part of the numerical solution.

Before presenting the solution results, we provide the typical values for the governing parameters $\chi$ and $\mathcal{R}$ corresponding to field applications. We utilise the technique described by Kanin et al. (2020) in which several dimensional parameters are varied independently according to their representative value ranges while other are fixed. The field domains of the parameters are taken from (Kanin et al., 2020) with the addition of the value for the HF fluid density $\rho=10^{3} \mathrm{~kg} / \mathrm{m}^{3}$. Further, we compute the field domains of the dimensionless parameters $\mathcal{R}$ and $\chi$ in the parametric space $(\chi, \mathcal{R})$, and it has approximately the rectangular shape with the following boundaries: $\mathcal{R} \in(0.1,320.2)$ and $\chi \in(0.007,2032.4)$. It is also possible to highlight that the rock toughness $K_{I c}$ has the largest impact (among other parameters) on the boundary values of the characteristic Reynolds number $\mathcal{R}$, while the reservoir permeability $k$ defines the leak-off number $\chi$ variations.

\subsection{Examples of the general solution}

In this part of the paper, we present the numerically calculated fracture opening $\Omega$ and net fluid $\Pi$ pressure profiles for various values of the governing parameters $\chi$ and $\mathcal{R}$. We begin with the 
discussion of the case in which the Carter's leak-off number equals to $\chi=500$ and the characteristic Reynolds number is $\mathcal{R}=100$.

Figure 3 shows the obtained results. Fracture opening and net fluid pressure profiles in the $m k$ scaling are presented in (a) and (b), while these characteristics normalised by the far-field asymptote ( $t$-vertex) are depicted in (c) and (d). Apart from the solution with leak-off (solid black line), we also plot the corresponding profiles with zero leak-off (dashed black line). For comparison purposes, we show the laminar solution with leak-off by a dotted black line. In addition, in figures 3 (c) and (d), we also present the asymptotic regimes by coloured dashed lines.
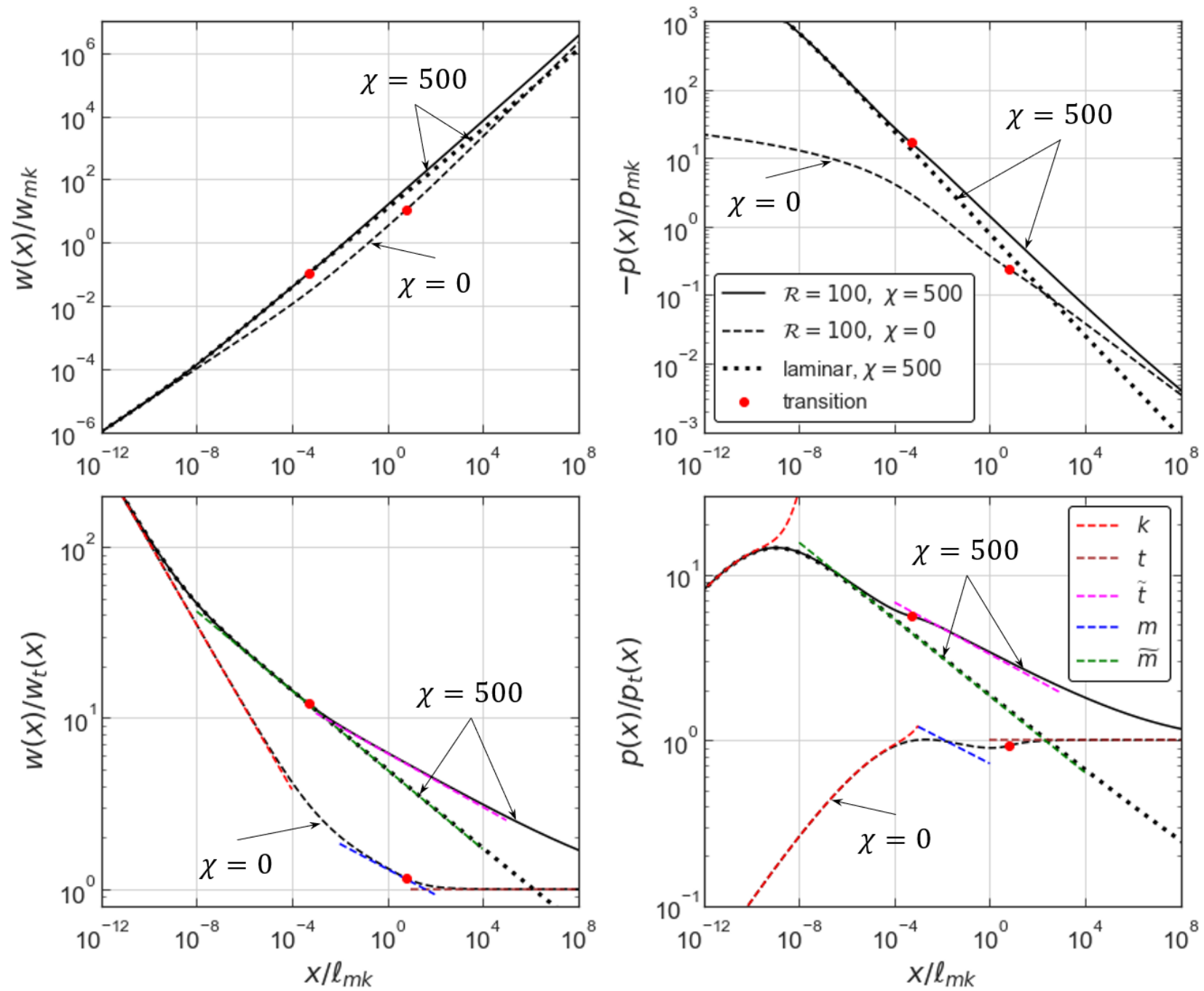

Figure 3: Solution for the fracture opening (a) and net fluid pressure (b) is shown in the $m k$-scaling for $\chi=500$ and $\mathcal{R}=100$. These characteristics normalised by $t$-vertex solution are presented in (c) and (d). The corresponding solution without leak-off $(\chi=0)$ is depicted by a dashed black line. The laminar solution with leak-off $(\chi=500)$ is presented by a dotted black line. In (c) and (d), different vertex solutions are depicted by coloured dashed lines. In (d), the $k$ asymptotic expansion (red dashed line) is plotted twice: for both solutions (with and without leak-off) individually. By red circle dots, we mark the locations of laminar-to-turbulent transition points for laminar-turbulent solutions with and without leak-off.

Let us look at the opening profiles. Using the numerical calculations accounting for the laminarto-turbulent transition, we can notice that both profiles (with and without leak-off) have the same asymptotic behaviour in the near- (red dashed line) and far-field (brown dashed line) governing by $k$ and $t$-vertex solutions, respectively. From figure $3(\mathrm{c})$, one can find out that the applicability domains 
of the toughness and turbulent-storage-viscosity regimes are much larger for $\chi=0$ than for the case with $\chi=500$ implying shrinkage of storage dominated domains with increasing leak-off number $\chi$. Two considered solutions differ significantly in the intermediate-field, namely, the fracture aperture is larger for all distances $\xi$ in the shown coordinate range for the non-zero leak-off case. When $\chi=0$, we observe the laminar-storage-viscosity $(m)$ asymptote in the intermediate-field (blue dashed line) which is in the agreement with results of Lecampion and Zia (2019). In turn, in the intermediate-field of the solution with $\chi=500$, we obtain two different limiting regimes: laminar-leak-off-viscosity $(\tilde{m})$ closer to the tip and then turbulent-leak-off-viscosity $(\tilde{t})$ depicted by green and magenta dashed lines, respectively. Using figure 3, one can also determine that the laminar-to-turbulent transition point for the solution with leak-off is located much closer to the moving fracture tip compared to the solution with zero leak-off $\left(\Lambda(500,100) \approx 5 \cdot 10^{-4}\right.$ and $\left.\Lambda(0,100) \approx 6.7\right)$. Comparing the opening profiles of the purely laminar $\left(n=1, f_{0}^{\prime \prime}=1\right)$ and laminar-turbulent $(\mathcal{R}=100)$ fracture solutions with leak-off $(\chi=500)$, one can observe that they coincide in the laminar flow region $(\xi<\Lambda(500,100))$, while the opening in the laminar-turbulent fracture exceeds that in the purely laminar solution past the transition point $\xi>\Lambda(500,100)$. In the intermediate-field of the laminar solution with leak-off, the laminar-leak-off-viscosity regime $(\tilde{m})$ is realised (figure $3(\mathrm{c})$ ).

Further, we move on to the net pressure profiles (figures $3(b)$ and (d)). One can observe that the pressure solutions with $\mathcal{R}=100$ for Carter's leak-off and impermeable reservoir cases are different in the near and intermediate-fields, and they have the same $t$-asymptote in the far-field. Since the net pressure is zero for $k$-vertex, we utilise the next-order term of the asymptotic expansion (Garagash et al., 2011) which depends on $\chi$ (in this step, we assume that the $k$-vertex is located inside the region with laminar flow). As a result, there are two different red dashed lines in figure 3 (d) corresponding to respective value of the leak-off parameter. From this figure, it can also be noticed that the appearance of the intermediate asymptotes $(m, \tilde{m}$ and $\tilde{t})$, and their applicability domains are smaller as compared to those for the crack aperture. Similar to the opening profiles, we notice that the pressure profiles for the laminar and turbulent cases with leak-off are the same along the zone with laminar flow but its length is much smaller than $\Lambda: \xi<10^{-6}$.

Dependence of the problem solution on the governing parameters $\chi$ and $\mathcal{R}$ is further explored in figure 4. In figure 4(a), we exemplify how the crack opening profile varies with the leak-off inten-
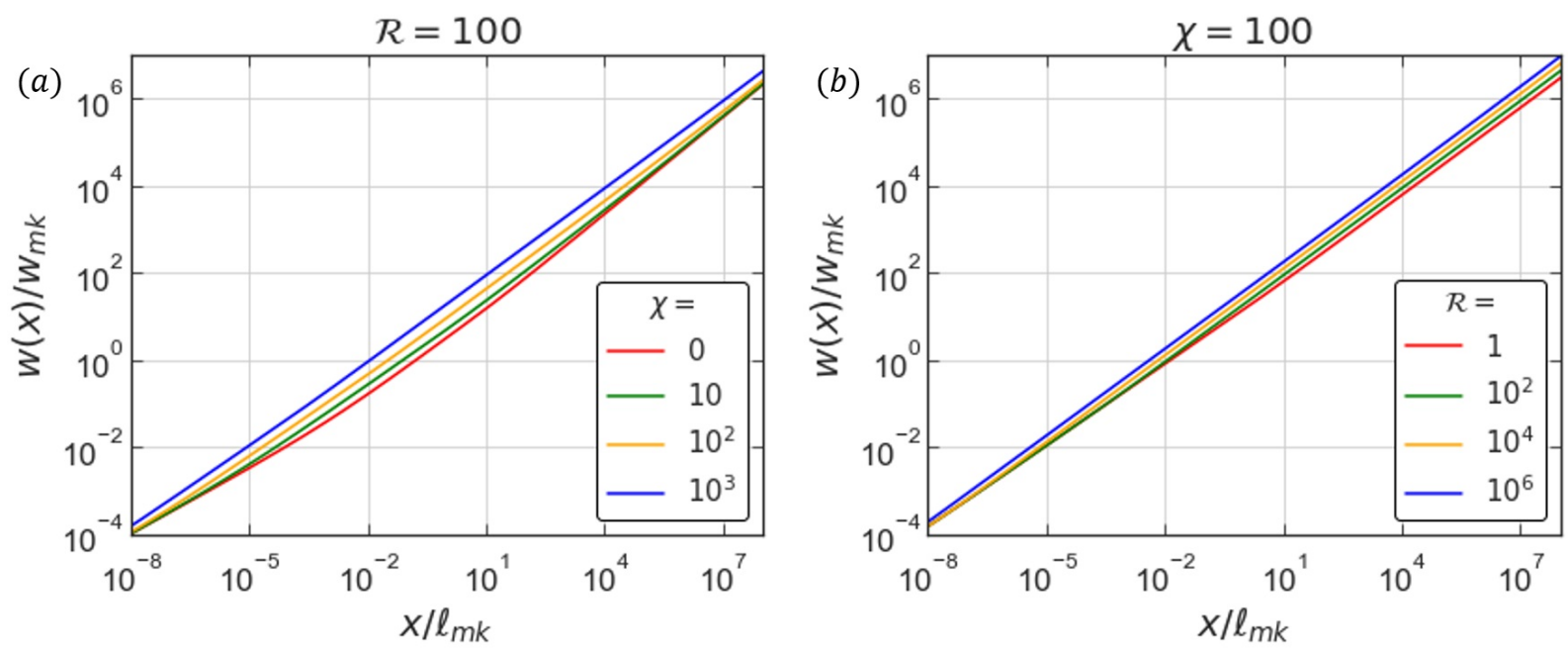

Figure 4: Solutions for the fracture opening in the $m k$-scaling corresponding to (a): $\mathcal{R}=100$ and $\chi=0,10,10^{2}, 10^{3}$ and (b): $\chi=100$ and $\mathcal{R}=1,10^{2}, 10^{4}, 10^{6}$.

sity $\chi$ while the characteristic Reynolds number value is fixed. One can find out that all profiles start from the near-field asymptote, and, in the far-field, they approach the $t$-vertex solution. In the 
intermediate-field, the crack aperture is seen to increase with the Carter's number $\chi$. Figure 4(b) examines the dependence of the fracture aperture on Reynolds number at a fixed leak-off value. The profiles have the same near-field and different far-field asymptotic behaviour since $\Omega_{t} \sim \mathcal{R}^{(1-n) /(n+2)}$ in the latter, i.e. higher values of the characteristic Reynolds number $\mathcal{R}$ lead to larger aperture in the far-field. Overall, and across scales, figures $4(a)$ and (b) illustrate the increase of the fracture opening with increase of either leak-off $(\chi)$ or turbulence $(\mathcal{R})$ intensities.

\section{Discussion}

\subsection{Examination of the transition length}

In this section, we determine how the laminar-to-turbulent transition length depends on the governing parameters which is expressed in the $m k$-scaling as $\Lambda(\chi, \mathcal{R})$. This characteristic distance is a solution of the non-linear equation (29), and we calculate it from the numerical solution for crack opening profile.

When we introduce the leak-off process into the tip model, we effectively increase the fluid flow velocity $v(x)$ by the value of Carter's term, and Reynolds number becomes higher than the critical value $R e_{c}$ much closer to the moving tip. This concept is confirmed by the numerical results presented in the previous section in which $\Lambda(500,100)=5 \cdot 10^{-4}$ and $\Lambda(0,100)=6.7$ indicating that the crack region with the laminar flow regime in the case of $\chi>0$ has smaller size as compared to an impermeable reservoir case, or, in other words, the turbulent flow regime occupies the wider fracture domain.

Further, we move on to the investigation the whole parametric space $(\chi, \mathcal{R})$. In figure $5(\mathrm{a})$, we present the colour map with the values of $\Lambda(\chi, \mathcal{R})$ function. In turn, figure 5(b) shows the dependence the transition length on the leak-off number when the characteristic Reynolds number is constant, and figure 5(c) depicts the opposite situation ( $\chi$ is constant, and $\mathcal{R}$ varies). In other worlds, figures $5(\mathrm{~b})$ and (c) are slices of figure 5(a) along $\chi(\mathrm{OX})$ and $\mathcal{R}$ (OY) axes, correspondingly. Using figures $5(a)$ and (b), one can notice that for each $\mathcal{R}$, the transition length remains constant until certain value of $\chi$, i.e. the leak-off does not impact the flow regime transition until some threshold value of $\chi$. If we continue to increase the leak-off number $\chi$, the transition length eventually decreases, meaning that the fracture domain with the laminar flow regime shrinks. Looking at the profiles depicted in figure 5 (c), we find out that the transition length is a decaying function on the characteristic Reynolds at fixed leak-off. We also determine that the transition length is closely approximated by $\Lambda \sim R e_{c}^{2}(\mathcal{R} \chi)^{-2}$ for $\chi \gg 1$ stemming from neglecting the storage term, i.e. $\Omega(\Lambda)$, compared to the leak-off in (29) (coloured dashed lines in figure $5(\mathrm{~b})$ ). It is also possible to notice that $\Lambda(0, \mathcal{R})$ (constant values in figure $5(\mathrm{~b})$ for small $\chi$ or red curve in figure $5(\mathrm{c})$ ) can be approximated by $\Lambda \sim \operatorname{Re}_{c}^{3 / 2}\left(\beta_{0} \mathcal{R}\right)^{-3 / 2}=6826 \mathcal{R}^{-3 / 2}$ for $\mathcal{R}<10^{4}$ (dashed red line in figure $5(\mathrm{c})$ ). This result is obtained by neglecting the leak-off term in equation (29) and substituting $m$-vertex solution for the opening. Lecampion and Zia (2019) utilise this power law, i.e. $\sim \mathcal{R}^{-3 / 2}$, to fit the transition length $\Lambda(0, \mathcal{R})$ in the whole range of the characteristic Reynolds numbers in their zero-leak-off solution and obtain $\Lambda \sim 5000 \mathcal{R}^{-3 / 2}$.

\subsection{Applicability domains of the vertex solutions}

In the present section, we consider spatial domains where the general numerical solution can be approximated by the limiting (vertex) solutions. Similar to Garagash et al. (2011), we define an asymptotic bound as a distance from the moving tip at which the crack opening profile deviates from the considered vertex solution (Table 1 and equations (17), (19)) by $1 \%$. Let us denote the upper bound of 

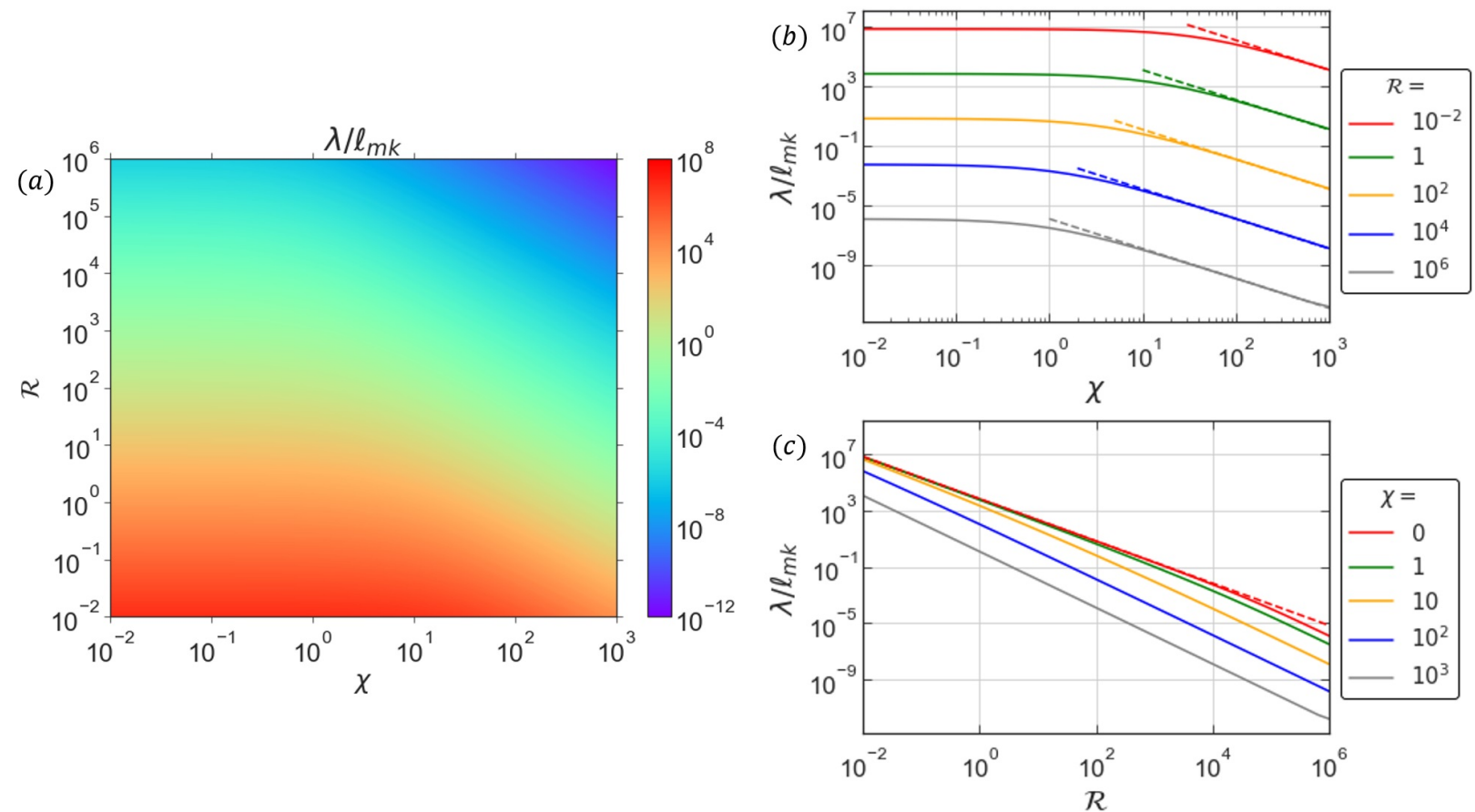

Figure 5: Transition length (i.e. distance from the moving tip where the laminar flow regime transforms to the turbulent one) depending on the leak-off $\chi$ and characteristic Reynolds $\mathcal{R}$ numbers (equation (29)). The colour map with $\Lambda(\chi, \mathcal{R})$ values is presented in (a). Several cross-section of (a) figure are shown in: (b), where the characteristic Reynolds number is fixed, and (c), in which the leak-off coefficient is constant. Using coloured dashed lines, we present analytical asymptotes for $\Lambda(\chi \gg 1, \mathcal{R}=$ const $)$ in (b) and for $\Lambda(0, \mathcal{R})$ in (c).

the $k$-vertex asymptotic domain by $x_{k}$, i.e. this regime is located inside the interval $x \in\left(0, x_{k}\right)$, and by $x_{t}$, we define the lower bound of the $t$-vertex asymptotic region: $x \in\left(x_{t},+\infty\right)$. In the same way, one can define the domains of the vertices that realise in the intermediate field: $x \in\left(x_{m}^{0}, x_{m}^{\infty}\right)$ for the $m$-vertex, $x \in\left(x_{\tilde{m}}^{0}, x_{\tilde{m}}^{\infty}\right)$ for the $\tilde{m}$-vertex, $x \in\left(x_{\tilde{t}}^{0}, x_{\tilde{t}}^{\infty}\right)$ for the $\tilde{t}$-vertex. Similar to Kanin et al. (2020), we define the storage domain boundary $x_{S}=\xi_{S} \ell_{m k}: \Omega\left(\xi_{S}\right)=\chi \sqrt{\xi_{S}} / 0.05$ such that the cumulative leaked-off volume is a small fraction (5\%) of the fracture storage when $x>x_{S}$. Since the approximation of the MDR asymptote (equation (11)) has limited applicability domain ( $R e<1.5 \cdot 10^{4}$ ), we also introduce the boundary $x_{B}=\xi_{B} \ell_{m k}$ connected with this limit: $\Omega\left(\xi_{B}\right)+\chi \sqrt{x_{B}}=1.5 \cdot 10^{4} / \mathcal{R}$ which defines the validity region $x<x_{B}$ of our approximation of the slickwater turbulent behaviour.

Figure 6 shows the regime maps in the parametric space $(\chi, \mathcal{R})$ for $\chi=0,0.1,1,10,50,100,500$ and 1000 . By coloured lines, we present the domain boundaries of the vertex solutions, the laminarto-turbulent transition length is shown by black dashed line; $x_{S}$ and $x_{B}$ lengths are depicted by dashed-dotted and dotted lines, respectively.

Figure 6(a) presents the zero leak-off case, and the results are in agreement with the regime map constructed by Lecampion and Zia (2019). Let us consider the near-field $(k)$ and far-field $(t)$ asymptotes first. Based on figures 6(a)-(h), one can observe that $\xi_{k}=x_{k} / \ell_{m k}$ (red lines) is a function of the leak-off number only (in the considered range of $\mathcal{R}$ ), i.e. the characteristic Reynolds number does not affect this limiting solution, and $\xi_{k}(\xi)$ coincides with that of Garagash et al. (2011) for the laminar flow regime. Such observation can be explained by the fact that $k$-vertex is always realised in the crack region with laminar flow within the parametric region shown in the figure 6; however, for large enough $\mathcal{R}>10^{6}$ (not shown), the upper boundary of the $k$-vertex skirts the turbulent zone and is expected to depend on the value of the characteristic Reynolds number there. The near-field asymptotic domain shrinks with increase in the leak-off intensity, i.e. $x_{k}$ moves towards the fracture 


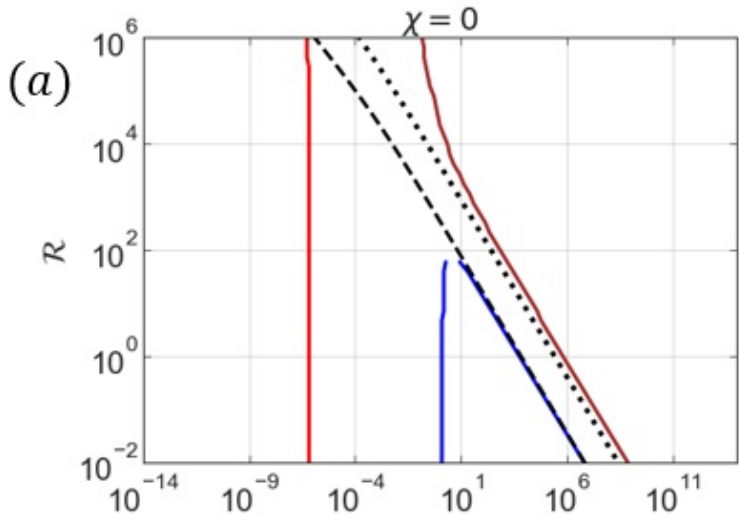

(b)

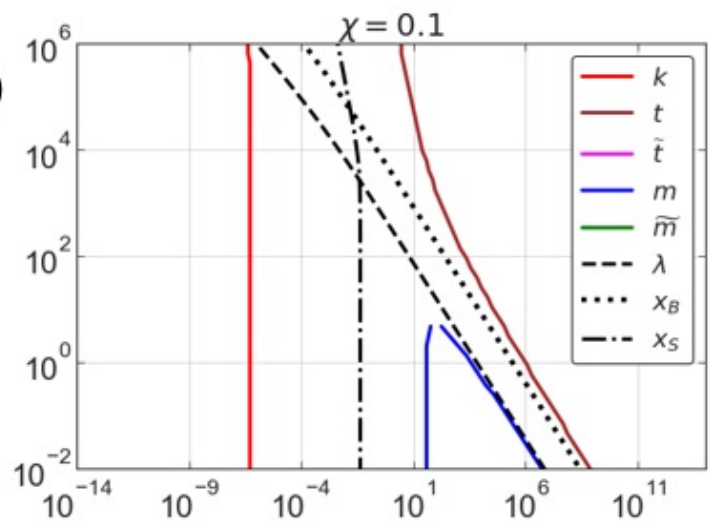

(c)

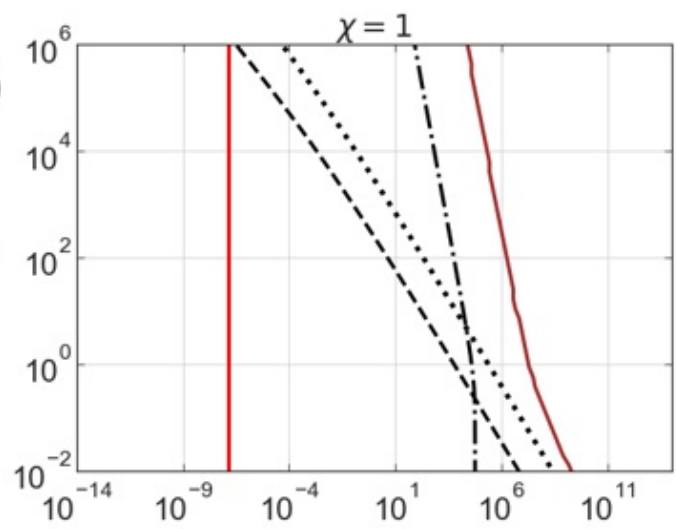

(d)

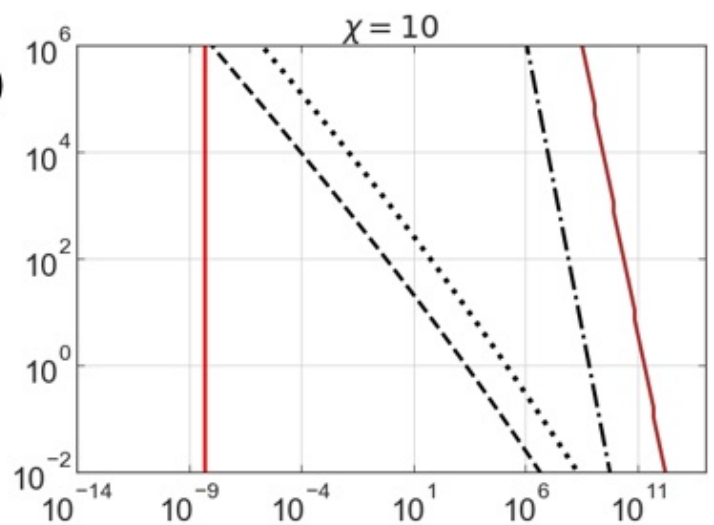

(e)

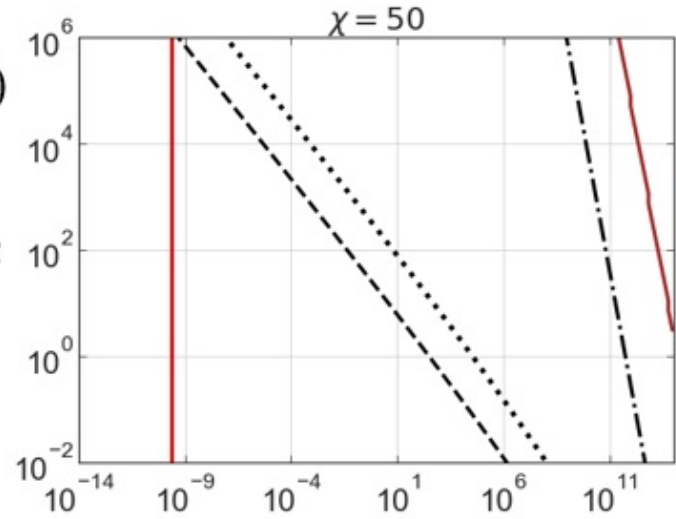

$(f)$
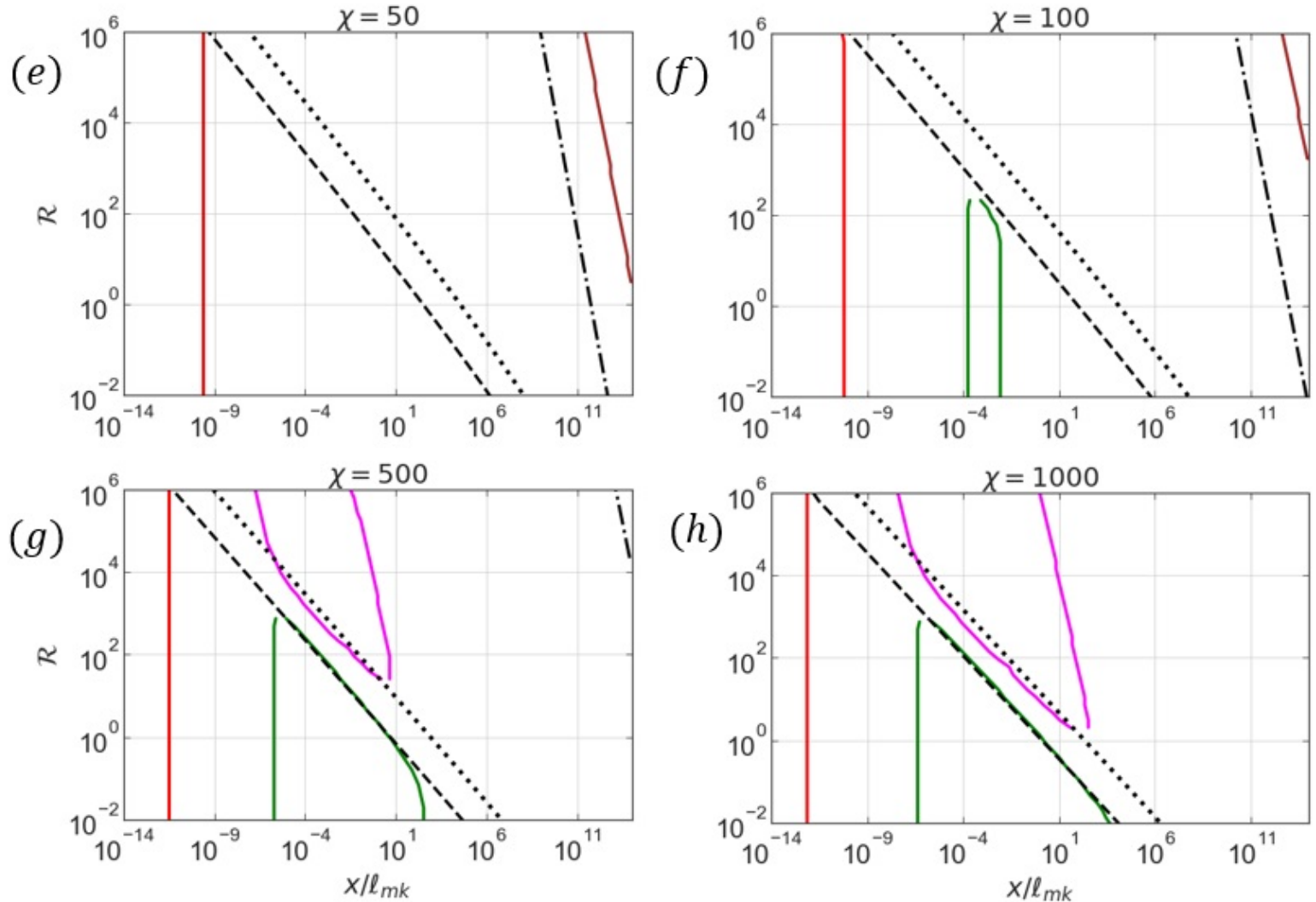

Figure 6: Regime maps presenting the boundaries of the spatial domains corresponding to the various limiting propagation regimes (vertex solutions) (Table 1 and equations (17), (19)) in coordinates $\left(x / \ell_{m k}, \mathcal{R}\right)$ for various values of $\chi$. We show the laminar-to-turbulent transition length $\lambda$ by a dashed line, the storage domain boundary $x_{S}$ by a dashed-dotted line and the applicability limit of the approximation of the MDR asymptote $x_{B}$ by a dotted line. 
tip. In turn, the lower bound of the $t$-vertex domain (brown lines) moves away from the tip when $\chi$ grows (in figures $6(\mathrm{~g})$ and $(\mathrm{h})$, it is out of the computational domain). The boundary $\xi_{t}=x_{t} / \ell_{m k}$ is a non-linear function on both governing parameters. In figures $6(a)$ and (b), we find out the presence of the laminar-storage-viscosity $(m)$ regime (blue line), it can be noticed for $\chi<0.5$ for the chosen range of the characteristic Reynolds number. Starting from $\chi=80$, we observe the $\tilde{m}$-vertex in the intermediate-field (figures 6(f)-(g), green line), and for the leak-off number values $\chi>120$, the turbulent-leak-off-viscosity $(\tilde{t})$ regime also approximates the general numerical solution (magenta line). When we increase $\chi$ value, the asymptotic domains of $\tilde{m}$ and $\tilde{t}$ expands.

Let us move on to the discussion of the characteristic boundaries presented in figure 6 . The transition boundary between the laminar and turbulent flow regimes $(\lambda)$ is shown by dashed black line. Based on the results, we find out that the crack zone occupied by laminar flow decreases when the leak-off number $\chi$ grows, i.e. the transition boundary moves closer to the fracture front. As a result, in the permeable reservoir case, the turbulent flow regime has a more significant impact on the crack tip characteristics since it is realised on the larger part of a semi-infinite fracture as compared to the zero-leak-off case (Lecampion and Zia, 2019). By dotted-dashed line, we present the boundary of the crack-storage-domain $\left(x_{S}\right)$ which is on the right-hand side of this line $\left(x>x_{S}\right)$. The leak-off process can be neglected in this region, and from figure 6, we observe that the crack-storage-domain shrinks ( $x_{S}$ migrates away from the tip) with $\chi$ growth. The final boundary $\left(x_{B}\right)$ is marked by dotted line, and it depicts the upper limit of the applicability region of the MDR asymptote approximation (11). One can remark that the proposed near-tip solution is correct along the spatial region $x \in\left(0, x_{B}\right)$, and for larger distances from the tip (i.e. $x>x_{B}$ ), it provides a semi-infinite fracture characteristics corresponding to the fluid flow inside the crack channel with an underestimated friction (figure 2). From figure 6 , we notice that $x_{B}$ boundary is parallel (in double logarithmic scale) to the transition length, and the size of the crack domain with the 'correct' solution diminishes with increasing leakoff.

Further, we determine the orientation of various boundaries of the asymptotic domains in figure 6 using analytical considerations, i.e. we approximate the boundaries using power law function $\mathcal{R} \sim \xi^{\alpha}$ and calculate the exponents $\alpha$ (the proportionality coefficients can be estimated from the numerical solution). It is known that the boundaries orientation is governed by the characteristic length scales of the transitions between various limiting propagation regimes. In figures $6(\mathrm{a})$ and (b), the boundary $x_{m}^{0}$ is located inside the region with laminar flow meaning, and it does not depend on $\mathcal{R}$ (vertical line). The same explanation can be applied for the $\tilde{m}$-vertex boundaries (figures $6(\mathrm{f})-(\mathrm{h})$ ): $x_{\tilde{m}}^{0}$ and $x_{\tilde{m}}^{\infty}$ (segments below the transition boundary). Let us consider the boundaries $x_{m}^{\infty}$ and $x_{t}$ (segments: $\mathcal{R}<100$ for $\chi=0$ and 0.1 ; $\mathcal{R}<1$ for $\chi=1$ ), and they have the certain inclination angle which we determine according to the following derivations. The lengthscale $\ell_{t m}$ which characterises the $t m$-transition can be found from the comparison of the opening profiles: $w_{m}\left(\ell_{t m}\right) \sim w_{t}\left(\ell_{t m}\right)$, and it is proportional to $\ell_{t m} \sim \mathcal{R}^{-3 / 2} \ell_{m k}$. Such transitions between vertices are also called as edgesolutions (Garagash et al., 2011). Each considered boundary corresponds to constant value of $x / \ell_{t m}$, and these constants can be found from the numerical solution. Using the determined expression for the length scale $\ell_{t m}$, we obtain that the boundaries $x_{m}^{\infty}$ and $x_{t}$ ( $\mathcal{R}<100$ for $\chi=0$ and $0.1 ; \mathcal{R}<1$ for $\chi=1$ ) are described by: $\mathcal{R} \sim \xi^{-2 / 3}$. Further, we consider the $t k$-transition, and the characteristic length scale in this case is $\ell_{t k} \sim \mathcal{R}^{(2 n-2) /(2-n)} \ell_{m k}$. Using this formula and the calculations, we determine that the boundary $x_{t}\left(\mathcal{R}>10^{4}\right.$ for $\chi=0$ and $0.1 ; \mathcal{R}>100$ for $\chi=1$; $\left.\chi=10,50,100\right)$ corresponds to certain constant $x / \ell_{t k}$, and it is governed by the equation $\mathcal{R} \sim \xi^{-(2-n) /(2-2 n)}$. Furthermore, it is possible to assume that $x_{k}$ boundary is also have this form when it is located inside the region with the turbulent flow regime, e.g. for $\mathcal{R}>10^{6}$ and $\chi=0, \ldots, 1000$. Further, we move on to the transition $\tilde{t} k$, and the characteristic length scale is proportional to $\ell_{\tilde{t} k} \sim \mathcal{R}^{(2 n-2) /(2-n)} \chi^{-2} \ell_{m k}$. As a result, the boundary $x_{\tilde{t}}^{0}$ for $\mathcal{R}>10^{4}$ (figure $6(\mathrm{~g})$ and $(\mathrm{h})$ ) has the form: $\mathcal{R} \sim \xi^{-(2-n) /(2-2 n)}(\chi$ is fixed on each map and is not accounted in the formula). Moreover, if we consider the $t \tilde{t}$-edge, we 
obtain $\ell_{t \tilde{t}} \sim \mathcal{R}^{(2 n-2) /(2-n)} \chi^{-2(n+2) /(2-n)} \ell_{m k}$ meaning that $x_{\tilde{t}}^{\infty}$ is parallel the boundary $x_{\tilde{t}}^{0}\left(\mathcal{R}>10^{4}\right)$. Next, we can derive the inclination angle related to the boundaries $x_{\tilde{t}}^{0}\left(\mathcal{R}<10^{4}\right.$ ) and $x_{\tilde{m}}^{\infty}$ (the segment which coincides with the transition length). For its determination, we look at the $\tilde{t} \tilde{m}$-transition whose length scale is $\ell_{\tilde{t} \tilde{m}} \sim \mathcal{R}^{-2} \chi^{-2} \ell_{m k}$. By taking into account that each considered boundary corresponds to constant value of $x / \ell_{\tilde{t} \tilde{m}}$, we obtain: $\mathcal{R} \sim \xi^{-1 / 2}$.

\section{Conclusions}

In this paper, we use a steady-state model of a semi-infinite fracture moving with a constant velocity to analyse the near-tip region of a hydraulic fracture propagating in a permeable reservoir. In the considered model, we account for a possible transition of the fluid flow regime from laminar to turbulent, moving away from the fracture tip. The hydraulic fracturing fluid is taken as slickwater which frictional properties are governed by an approximate form (Lecampion and Zia, 2019) of the maximum drag reduction (MDR) (Virk, 1971, 1975) asymptote. The fluid exchange process between the fracture and the ambient rock is implemented in Carter's leak-off form. We carry out normalisation of the governing equations and determine that the problem solution (crack opening and net fluid pressure profiles) depends on two dimensionless parameters (apart from the scaled distance from the tip): Carter's leak-off number $\chi=2 C^{\prime} E^{\prime} /\left(\sqrt{V} K^{\prime}\right)$ and characteristic Reynolds number $\mathcal{R}=12 \rho K^{\prime 4} /\left(E^{\prime 3} \mu^{\prime 2}\right)$.

We apply the numerical method of Garagash et al. (2011) to find the general solution and explore its behaviour in the problem parametric space. We enumerate all limiting regimes (also known as vertices) realised in the model and plot regime maps to depict their spatial applicability domains in relation to the general solution. We also focus on the laminar-to-turbulent transition boundary between the flow regimes as it indicates the potential importance of this transformation to the fracture solution. Based on the result, we can conclude that the fracture region with the laminar flow regime shrinks towards the tip with an increase of the leak-off number $\chi$ (while $\mathcal{R}$ is fixed) as compared to the zero leak-off case (Lecampion and Zia, 2019). As a result, turbulence has a greater impact on the fracture tip solution when the fracturing fluid leak-off is present (while the rest of the problem parameters and the propagation velocity are kept the same).

The present fracture tip model allows one to accurately resolve the interplay between turbulent and laminar flow regimes inside the fracture channel, leak-off process, and brittle rock failure near the moving front. Moreover, the tip model helps to capture the combined effects of these processes on the transient propagation of a finite fracture. If it is necessary to investigate the finite fracture growth with leak-off and turbulent flow, the proposed model can be implemented as a tip element which determines the fracture front velocity at each time step by matching the fracture opening from the global numerical solution and local near-tip asymptotic behaviour at the spatial segment adjusted to the tip element (Peirce and Detournay, 2008; Peirce, 2015; Dontsov, 2016a; Dontsov and Peirce, 2017; Zia and Lecampion, 2019).

\section{Acknowledgements}

Startup funds of Skolkovo Institute of Science and Technology are gratefully appreciated by A.A. Osiptsov and E.A. Kanin. Support for this research by the Natural Science and Engineering Research Council of Canada (Discovery grant 05743 to D.I. Garagash) is gratefully acknowledged. 


\section{References}

Adachi, J. I. and Detournay, E. (2008). Plane strain propagation of a hydraulic fracture in a permeable rock. Engineering Fracture Mechanics, 75(16):4666-4694.

Batchelor, G. K. (1967). An Introduction to Fluid Dynamics. Cambridge University Press, Cambridge UK.

Bessmertnykh, A. O. and Dontsov, E. V. (2019). A semi-infinite hydraulic fracture driven by a herschelbulkley fluid. Journal of Applied Mechanics, 86(12).

Bilby, B. and Eshelby, J. (1968). Dislocations and the theory of fracture. In Liebowitz, H., editor, Fracture, an Advanced Treatise, volume I, chapter 2, pages 99-182. Academic Press, New York NY.

Brill, J. P. and Mukherjee, H. K. (1999). Multiphase flow in wells, volume 17. Society of Petroleum Engineers.

Bunger, A. P., Detournay, E., and Garagash, D. I. (2005). Toughness-dominated hydraulic fracture with leak-off. International journal of fracture, 134(2):175-190.

Carter, E. (1957). Optimum fluid characteristics for fracture extension. In Howard, G. C. and Fast, C. R., editors, Drilling and Production Practices, pages 261-270.

Desroches, J., Detournay, E., Lenoach, B., Papanastasiou, P., Pearson, J. R. A., Thiercelin, M., and Cheng, A. (1994). The crack tip region in hydraulic fracturing. Proceedings of the Royal Society of London. Series A: Mathematical and Physical Sciences, 447(1929):39-48.

Detournay, E. (2016). Mechanics of hydraulic fractures. Annual Review of Fluid Mechanics, 48:311339.

Detournay, E. and Garagash, D. (2003). The near-tip region of a fluid-driven fracture propagating in a permeable elastic solid. Journal of Fluid Mechanics, 494:1-32.

Dontsov, E. (2016a). An approximate solution for a penny-shaped hydraulic fracture that accounts for fracture toughness, fluid viscosity and leak-off. Royal Society open science, 3(12):160737.

Dontsov, E. (2016b). Tip region of a hydraulic fracture driven by a laminar-to-turbulent fluid flow. Journal of Fluid Mechanics, 797.

Dontsov, E. (2017). An approximate solution for a plane strain hydraulic fracture that accounts for fracture toughness, fluid viscosity, and leak-off. International Journal of Fracture, 205(2):221-237.

Dontsov, E. and Kresse, O. (2018). A semi-infinite hydraulic fracture with leak-off driven by a powerlaw fluid. Journal of Fluid Mechanics, 837:210-229.

Dontsov, E. and Peirce, A. (2017). A multiscale implicit level set algorithm (ilsa) to model hydraulic fracture propagation incorporating combined viscous, toughness, and leak-off asymptotics. Computer Methods in Applied Mechanics and Engineering, 313:53-84.

Economides, M., Oligney, R., and Valkó, P. (2002). Unified fracture design: bridging the gap between theory and practice. Orsa Press.

Economides, M. J., Nolte, K. G., et al. (1989). Reservoir stimulation, volume 2. Prentice Hall Englewood Cliffs, NJ.

Garagash, D. and Detournay, E. (2000). The tip region of a fluid-driven fracture in an elastic medium. J. Appl. Mech., 67(1):183-192.

Garagash, D. I. (2006). Plane-strain propagation of a fluid-driven fracture during injection and shut-in: Asymptotics of large toughness. Engineering fracture mechanics, 73(4):456-481. 
Garagash, D. I. (2019). Cohesive-zone effects in hydraulic fracture propagation. Journal of the Mechanics and Physics of Solids, 133:103727.

Garagash, D. I., Detournay, E., and Adachi, J. I. (2011). Multiscale tip asymptotics in hydraulic fracture with leak-off. J. Fluid Mech., 669:260-297, DOI: 10.1017/S002211201000501X.

Hu, J. and Garagash, D. (2010). Plane-strain propagation of a fluid-driven crack in a permeable rock with fracture toughness. Journal of Engineering Mechanics, 136(9):1152-1166.

Irvin, G. R. (1957). Analysis of stresses and strains near the end of a crack traversing a plate. ASME J. Appl. Mech., 24:361-364.

Kanin, E. A., Garagash, D. I., and Osiptsov, A. A. (2020). The near-tip region of a hydraulic fracture with pressure-dependent leak-off and leak-in. Journal of Fluid Mechanics, 892:A31, DOI: $10.1017 / \mathrm{jfm} .2020 .193$.

Kanninen, M. F. and Popelar, C. L. (1985). Advanced fracture mechanics.

Kovalyshen, Y. (2010). Fluid-driven fracture in poroelastic medium.

Lecampion, B. and Zia, H. (2019). Slickwater hydraulic fracture propagation: near-tip and radial geometry solutions. Journal of Fluid Mechanics, 880:514-550.

Lenoach, B. (1995). The crack tip solution for hydraulic fracturing in a permeable solid. Journal of the Mechanics and Physics of Solids, 43(7):1025-1043.

Lister, J. R. (1990). Buoyancy-driven fluid fracture: the effects of material toughness and of lowviscosity precursors. Journal of Fluid Mechanics, 210:263-280.

Madyarova, M. V. (2003). Fluid-driven penny-shaped fracture in elastic medium. PhD thesis, University of Minnesota.

Moukhtari, F.-E. and Lecampion, B. (2018). A semi-infinite hydraulic fracture driven by a shearthinning fluid. Journal of Fluid Mechanics, 838:573-605.

Nieuwstadt, F. T., Westerweel, J., and Boersma, B. J. (2016). Turbulence: introduction to theory and applications of turbulent flows. Springer.

Peirce, A. (2015). Modeling multi-scale processes in hydraulic fracture propagation using the implicit level set algorithm. Computer Methods in Applied Mechanics and Engineering, 283:881-908.

Peirce, A. and Detournay, E. (2008). An implicit level set method for modeling hydraulically driven fractures. Computer Methods in Applied Mechanics and Engineering, 197(33-40):2858-2885.

Rubin, A. M. (1993). Tensile fracture of rock at high confining pressure: implications for dike propagation. Journal of Geophysical Research: Solid Earth, 98(B9):15919-15935.

Savitski, A. and Detournay, E. (2002). Propagation of a penny-shaped fluid-driven fracture in an impermeable rock: asymptotic solutions. International journal of solids and structures, 39(26):63116337.

Spence, D. and Sharp, P. (1985). Self-similar solutions for elastohydrodynamic cavity flow. Proceedings of the Royal Society of London. A. Mathematical and Physical Sciences, 400(1819):289-313.

Virk, P. (1971). Drag reduction in rough pipes. Journal of fluid mechanics, 45(2):225-246.

Virk, P. S. (1975). Drag reduction fundamentals. AIChE Journal, 21(4):625-656.

Zia, H. and Lecampion, B. (2019). Pyfrac: A planar 3d hydraulic fracture simulator. arXiv preprint arXiv:1908.10788. 